International Journal of Pure and Applied Mathematics

Volume 102 No. 2 2015, 273-279

ISSN: 1311-8080 (printed version); ISSN: 1314-3395 (on-line version)

url: http://www.ijpam.eu

doi: http://dx.doi.org/10.12732/ijpam.v102i2.9

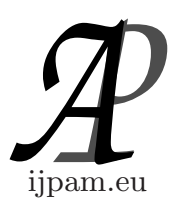

\title{
ON THE SEGRE UPPER BOUND OF THE REGULARITY FOR NON-FAT POINT SCHEMES IN PROJECTIVE SPACES
}

\author{
E. Ballico \\ Department of Mathematics \\ University of Trento \\ 38123 Povo (Trento) - Via Sommarive, 14, ITALY
}

\begin{abstract}
We study the generalized Segre bound in projective space (mainly in the plane) with respect to zero-dimensional schemes which are more general that the fat point schemes.
\end{abstract}

AMS Subject Classification: 13A02, 13D02, 14N05

Key Words: fat point, index of regularity, homogeneous ideal

$*$

Fix a finite set $S \subset \mathbb{P}^{n}$. For each $p \in S$ fix an integer $m_{p}>0$ and set $Z:=$ $\sum_{p \in S} m_{p} p$ (a fat point scheme). For any set $E \subseteq \mathbb{P}^{n}$ set $w(E):=\sum_{p \in S \cap E} m_{p}$. Fix an integer $d>0$. The generalized Segre condition says that $h^{1}\left(\mathcal{I}_{Z}(d)\right)=0$ (i.e. the zero-dimensional scheme $Z$ has index of regularity $\leq d$ ) if for all $c=1, \ldots, n$ and all $c$-dimensional linear spaces $A \subseteq \mathbb{P}^{n}$ we have $w(A) \leq c d+1$. The conditions for lines implies $m_{p} \leq d+1$ for all $p$ and that if $m_{p}=d+1$ for

Received: $\quad$ March 27, 2015

(c) 2015 Academic Publications, Ltd. url: www.acadpubl.eu 
some $p$, then $\sharp(S)=1$ and $h^{i}\left(\mathcal{I}_{Z}(d)\right)=0, i=0,1$. It is also easy to prove the generalized Segre conjecture if $m_{p}=d$ for some $p$. This conjecture is known only in some cases: if $S$ is in linearly general position ([5]), if $n \leq 3$ ([4], [6], [7], $[8])$, if $\sharp(S)=n+2$ and $S$ spans $\mathbb{P}^{n}([3])$ and if $\sharp(S)=n+3, S$ spans $\mathbb{P}^{n}$ and $Z$ is almost-equimultiple, i.e. $\left|m_{p}-m_{q}\right| \leq 1$ for all $p, q \in S$ ([9]). In this note we look at a similar conjecture for zero-dimensional schemes whose connected components are more general than fat points $m_{p} p$. Fix $P \in \mathbb{P}^{n}$, and integers $m>0$ and $e$ with $0 \leq e<\left(\begin{array}{c}m+n-1 \\ n-1\end{array}\right)$. The coherent sheaf $\left(\mathcal{I}_{P}\right)^{m} /\left(\mathcal{I}_{P}\right)^{m+1}$ is an $\left(\begin{array}{c}m+n-1 \\ n-1\end{array}\right)$-dimensional vector space over the algebraically closed base field. Let $\pi:\left(\mathcal{I}_{P}\right)^{m} \rightarrow\left(\mathcal{I}_{P}\right)^{m} /\left(\mathcal{I}_{P}\right)^{m+1}$ be the quotient map. Take a linear subspace $V \subseteq\left(\mathcal{I}_{P}\right)^{m} /\left(\mathcal{I}_{P}\right)^{m+1}$ with codimension $e$ and set $W:=\pi^{-1}(V)$. $W$ is a zerodimensional scheme, $m P \subseteq W \subsetneq(m+1) P$ and $\operatorname{deg}(W)=\operatorname{deg}(m P)+e$. We have $W=m P$ if and only if $e=0$. We say that $W$ has type $(m, e)$ and write $t_{W, p}=(m, e)$. The set $W(P, m, e)$ of all schemes of type $(m, e)$ with $P$ as their support is isomorphic to a Grassmannian and hence it is irreducible. Fix a finite set $S \subset \mathbb{P}^{n}$ For each $p \in S$ fix two integers $\left(k_{p}, e_{p}\right)$ with $k_{p}>0$ and $0 \leq e_{p}<\left(\begin{array}{c}k_{p}+n-1 \\ n-1\end{array}\right)$. Fix $W_{p} \in W\left(p, k_{p}, e_{p}\right), p \in S$, and set $Z:=\cup_{p \in S} W_{p}$. We say that $Z$ is tame if $e_{p} \leq 1$ for all $p \in S$. We say that $Z$ is general if $\left(W_{p}\right)_{p \in S}$ is general in $\prod_{p \in S} W\left(p, k_{p}, e_{p}\right)$. We say that $Z$ satisfies $\diamond$ if for all $p \in S$ we have $k_{p} \leq d+1$ and $e_{p}=0$ if $k_{p}=d+1$. Since $\operatorname{deg}\left(W_{p}\right)=\left(\begin{array}{c}n+k_{p}-1 \\ n\end{array}\right)+e$, condition $\diamond$ is a necessary condition to have $h^{1}\left(\mathcal{I}_{Z}(d)\right)=0$. For each $c=1, \ldots, n$, set $w(m, e, c):=m$ if $c+e \leq n$ and $w(m, e, c):=m+1$ if $e+c>n$. For any $c$-dimensional linear space $L \subseteq \mathbb{P}^{n}$ set $u_{Z}(L):=\sum_{p \in S \cap L} w\left(p, k_{p}, e_{p}\right)$. We say that $L$ satisfies the G-condition for $Z$ in degree $d$ or that $Z$ satisfies the G-condition at $L$ in degree $d$ if $u_{Z}(L) \leq c d+1$. We say that $Z$ satisfies the Gconditions in degree $d$ or that $(Z, d)$ satisfies the G-conditions if $Z$ satisfies $\diamond$ and it satisfies the G-condition in degree $d$ with respect to all positive dimensional linear subspaces of $\mathbb{P}^{n}$ (as in the case of the generalized Segre conditions we need to test also $\mathbb{P}^{n}$, i.e. the improper linear subspace).

Conjecture 1. Take $(Z, d)$ satisfying the $G$-conditions. Find additional condition which assure that $h^{1}\left(\mathcal{I}_{Z}(d)\right)=0$. We conjecture that $h^{1}\left(\mathcal{I}_{Z}(d)\right)=0$ if $Z$ is tame.

Theorem 1. Assume $n=2, Z$ tame and that the G-conditions are satisfied in degree $d$. Then $h^{1}\left(\mathcal{I}_{Z}(d)\right)=0$.

Then we consider the next step, when we weak the bound for $\mathbb{P}^{n}$, but we preserve the bounds for the lower dimensional linear spaces.

Theorem 2. Take $n=2$ and $Z, S, d$ such that $d \geq 4, \sharp(S) \geq 5, k_{p} \leq d$ for 
all $p$, the $G$-conditions are satisfied by all lines, but $u_{Z}\left(\mathbb{P}^{2}\right)=2 d+2$. Assume $h^{1}\left(\mathcal{I}_{Z}(d)\right)>0$. Then one of the following cases occur:

1. $e_{p}=0$ for all $p \in S$ and there is a conic $D$ with $S$ contained in the smooth locus of $D$;

2. $\sharp(S)=d+3, e_{q}=0$ for all $q \in S$, there is $p \in S$ with $k_{p}=d, k_{q}=1$ for all $q \notin S$ and no line through $p$ contains 3 points of $S$;

3. there is $p \in S$ with $t_{Z, p}=(d, 1), k_{q}=1$ for all $q \in S \backslash\{p\}$ and no line through $p$ contains 3 points of $S$.

The last condition in cases (2) and (3) of Theorem 2 is for free, because it comes from the G-condition for lines, which say that $\operatorname{deg}(L \cap Z) \leq d+1$ for every line $L$.

Remark 1. In the 3 cases listed we have $h^{1}\left(\mathcal{I}_{Z}(d)\right)>0$ for the following reasons. If $Z$ is a fat point scheme with $Z \subset D, D$ a reduced conic, then $\operatorname{deg}(Z) \geq 2 d+2>2 d+1=h^{0}\left(\mathcal{O}_{D}(d)\right)$. In the second and third case we have $\operatorname{deg}(Z)=\left(\begin{array}{c}d+2 \\ 2\end{array}\right)+1>h^{0}\left(\mathcal{O}_{\mathbb{P}^{2}}(d)\right)$ and hence $h^{1}\left(\mathcal{I}_{Z}(d)\right)>0$.

In [1] we introduced some conjectures and questions on the homogeneous ideal and the minimal free resolution of $Z$, up to a certain level. These questions may be translated into questions of the schemes introduced in this note using the function $u_{Z}(L)$, obvious generalizations of condition $\diamond$ and avoiding the cases $\left(k_{p}, e_{p}\right)$ with $k_{p}=d$, but we have no evidence for them.

Proof of Theorem 1: By [4] orr [7] we may assume that $Z$ is not a fat point scheme, i.e. the existence of $p \in S$ such that $a_{p}=\left(m_{p}, 1\right)$. For $i=0,1$ set $S_{i}:=\left\{p \in S: t_{p}=\left(m_{p}, i\right)\right.$. First assume $d=1$. By assumption $S_{1} \neq \emptyset$. We get $\sharp\left(S_{1}\right)=1$ and $\sharp\left(S_{0}\right) \leq 1$. It is sufficient to take $Z=v \cup e$ with $v$ a connected degree 2 scheme, say with support $p$, and with $e$ either $\emptyset$ or a point. If $e=\emptyset$, then $h^{1}\left(\mathcal{I}_{v}(1)\right)=0$ for any $v$. If $e$ is a point, then $h^{1}\left(\mathcal{I}_{Z}(1)\right)=0$ for a general $v$ with its support, i.e. for a $v$ such that the line spanned by $v$ does not contain $e$.

From now on we assume $d>1$ and that Theorem 1 is true for all positive integers $<d$. Since $\left.h_{A}^{1}(d)\right)=0$ if either $A=(d+1) p$ or $\left.A \in W(p, d, 1)\right)$, we may assume $\sharp(S) \geq 2$. Fix a line $L$ such that $u_{Z}(L)$ is maximal and set $B:=\operatorname{Res}_{L}(Z)$. If $Z$ is general, then $B$ is general with the restriction that $B_{\text {red }}$ is fixed and each point of $B_{\text {red }}$ has a type related to the one of $Z$. We have $B_{\text {red }} \subseteq S$. Fix $p \in S$ and let $Z_{p}$ be the connected component of $Z$ with $p$ as support. Set $B_{p}:=B \cap Z_{p}$. Set $\left(k_{p}, e_{p}\right):=t_{Z, p}$. If $p \notin L$, then $t_{B, p}=t_{Z, p}$. 
If $p \in L$, then $t_{B, p}=\left(k_{p}-1, e_{p}\right)$, except in the case $\left(k_{p}, e_{p}\right)=(1,1)$ in which $t_{B, p}=(1,0)$.

(i) Assume the existence of $p \in S$, such that $t_{B, p}=(d, 1)$. First assume $p \notin L$. We get $u_{Z}\left(\mathbb{P}^{2}\right) \geq d+1+u_{Z}(L)$. Hence $u_{Z}(L) \leq d$. Since $u_{Z}(L)>0$, there is $q \in L \cap S$. Let $D$ be the line spanned by $p$ and $q$. We get $u_{Z}(D) \geq$ $d+k_{q}>u_{Z}(L)$, contradicting the maximality property of $L$. Now assume $p \in L$. Since $d>1$, we get $\left(k_{p}, e_{p}\right)=(d+1,1)$ and so $Z$ does not satisfies $\diamond$, a contradiction. Hence $B$ satisfies condition $\diamond$.

(ii) We have $\operatorname{deg}(L \cap Z)=\sum_{p \in L \cap S} k_{p}$ and $\operatorname{deg}(B)=\operatorname{deg}(Z)-\operatorname{deg}(Z \cap L)$. The G-condition for $Z$ and $L$ is equivalent to $\operatorname{deg}(Z \cap L) \leq d+1$. Hence $h^{1}\left(L, \mathcal{I}_{Z \cap L}(d)\right)=0$. By the residual exact sequence

$$
0 \rightarrow \mathcal{I}_{B}(d-1) \rightarrow \mathcal{I}_{Z}(d) \rightarrow \mathcal{I}_{Z \cap L, L}(d) \rightarrow 0
$$

it is sufficient to prove that $B$ satisfies the G-conditions in degree $d-1$. We have $\sharp(S \cap L) \geq 2$, because $\sharp(S) \geq 2$. Note that $u_{B}\left(\mathbb{P}^{2}\right)=u_{Z}\left(\mathbb{P}^{2}\right)-\sharp(S \cap L)$ (even in the case in which $\left.\left(k_{p}, e_{p}\right)=(1,1)\right)$. Hence $u_{B}\left(\mathbb{P}^{2}\right) \leq 2(d-1)+1$. Fix a line $D \subset \mathbb{P}^{2}$. Since $u_{B}(D) \leq u_{Z}(D)$, to test the G-condition for all lines, it is sufficient to test it for the lines $D$ with $u_{Z}(D) \geq d+1$. The G-conditions for $Z$ give $u_{Z}(D)=d+1$. Since $u_{Z}(L) \geq u_{Z}(D)$, we get $u_{Z}(L)=d+1$. Assume for the moment $u_{B}(L)=d+1$. This is the case if and only if $\left(k_{p}, e_{p}\right)=$ $(1,1)$ for all $p \in S \cap L$. Since $u_{Z}(L)=d+1$, we get $u_{Z}\left(\mathbb{P}^{2}\right) \geq 2 d+2$, a contradiction. Now assume $D \neq L$ and set $\{p\}:=L \cup D$. If $p \notin S$, then we get $u_{Z}\left(\mathbb{P}^{2}\right) \geq u_{Z}(D)+w_{Z}(L)=2 d+2$, a contradiction. Hence $p \in S$. If $t_{Z, p}=\left(k_{p}, e_{p}\right) \neq(1,1)$, then $t_{B, p}=\left(k_{p}-1, e_{p}\right)$. If $t_{Z, p}=(1,1)$, then $t_{Z, p}=(1,0)$. In the latter case we get $w\left(\mathbb{P}^{2}\right) \geq u_{Z}(L)+u_{Z}(D) \geq 2 d+2$, a contradiction.

Proof of Theorem 2: The case in which $Z$ is a fat point scheme, i.e. $e_{p}=0$ is well-known (see [2] for much more or look at the proof of the general case below). Now assume $e_{p}>0$ for some $p \in S$. Take $\left(k_{p}, e_{p}\right), L$ and $B$ as in the proof of Theorem 1. Since $Z$ satisfies the G-conditions for all lines, we have $h^{1}\left(L, \mathcal{I}_{Z}(d)\right)=0$. The residual exact sequence $(1)$ gives $h^{1}\left(\mathcal{I}_{B}(d-1)\right)>0$. Note that $\sharp(S \cap L) \geq 2$.

(i) First assume $\sharp(S \cap L)=2$.

(i1) Assume the existence of a line $T$ such that $\sharp(S \cap T)>2$. Set $E:=$ $\operatorname{Res}_{T}(Z)$. For any $q \in S$ set $\left(m_{q}, g_{q}\right):=t_{E, q}$. If $q \notin T$, then $\left(m_{q}, g_{q}\right)=\left(k_{q}, e_{q}\right)$. Now assume $q \in T$. Since $T$ is a smooth curve, we have $\left(m_{q}, g_{q}\right)=\left(k_{q}-1, e_{q}\right)$ if $\left(k_{q}, e_{q}\right) \neq(1,1)$, and $\left(m_{q}, g_{q}\right)=(1,0)$ if $\left(k_{q}, e_{q}\right)=(1,1)$. We have $u_{E}\left(\mathbb{P}^{2}\right)=$ $u_{Z}\left(\mathbb{P}^{2}\right)-\sharp(S \cap T) \leq 2(d-1)+1$, i.e. $E$ satisfies the G-condition in degree $d-1$ with respect to $\mathbb{P}^{2}$. Since $k_{p} \leq d$ for all $p, E$ satisfies $\diamond$ in degree $d-1$, 
unless there is $p \in S$ with $\left(k_{p}, e_{p}\right)=(d, 1)$. Assume the existence of $p \in S$ with $\left(k_{p}, e_{p}\right)=(d, 1)$. Let $\ell: \mathbb{P}^{2} \backslash \mathbb{P}^{1}$ denote the linear projection from $p$. Let $Z_{p}$ be the connected component of $Z$ containing $p$. We have $h^{1}\left(\mathcal{I}_{Z_{p}}(d)\right)=0$ and $h^{0}\left(\mathcal{I}_{Z_{p}}(d)\right)=d$. Set $Z^{\prime}:=Z \backslash Z_{p}$ and $S^{\prime}:=S \backslash\{p\}$. Since $Z$ satisfies the G-conditions for lines, we have $\operatorname{deg}(D \cap Z) \leq d+1$ for all lines. Hence $k_{q}=1$ for all $q \neq p$ (i.e. $Z^{\prime}$ is a union of points and tangent vectors) and no line through $p$ contains 3 points of $S$. We are as in case (3).

(i2) Assume $\sharp(S \cap T)=2$ for all lines $T$. Since $\sharp(S) \geq 5$ and no 3 of the points of $S$ are collinear, there is a smooth conic $D \subset \mathbb{P}^{2}$ such that $\sharp(D \cap S) \geq 5$. We may find $D$ with the additional condition that it contains 4 points of $Z$ with maximal multiplicity., i.e. calling $m_{1} \geq \cdots \geq m_{s}, s=\sharp(S)$, the multiplicities of the points of $S$ in $Z$, we assume the existence of $p_{1}, p_{2}, p_{3}, p_{4}, p_{5} \in S \cap D$ such that $p_{i} \neq p_{j}$ for all $i \neq j$ and $k_{p_{i}}=m_{i}$ for all $i$. We have $h^{1}\left(D, \mathcal{I}_{D \cap Z}(d)\right)=0$ if and only if $\operatorname{deg}(D \cap Z) \geq 2 d+2$. Since $D$ is a smooth curve and $Z$ is general, this is the case if and only if $\sum_{q \in S \cap D} k_{q} \geq 2 d+2$. Since $u_{Z}\left(\mathbb{P}^{2}\right)=2 d+2$ we get $S \subset D$ and $e_{q}=0$ for all $q \in S$, i.e. we are in case (2) of Theorem 2 . Set $A:=\operatorname{Res}_{D}(Z)$. For any $q \in S$ set $\left(n_{q}, f_{q}\right):=t_{A, q}$. If $q \notin D$, then $\left(n_{q}, f_{q}\right)=\left(k_{q}, e_{q}\right)$. Now assume $q \in D$. Since $D$ is a smooth curve, we have $\left(n_{q}, f_{q}\right)=\left(k_{q}-1, e_{q}\right)$ if $\left(k_{q}, e_{q}\right) \neq(1,1)$, and $\left(n_{q}, f_{q}\right)=(1,0)$ if $\left(k_{q}, e_{q}\right)=(1,1)$. We get $u_{A}\left(\mathbb{P}^{2}\right) \leq u_{Z}\left(\mathbb{P}^{2}\right)-\sharp(S \cap D) \leq 2(d-2)+1$. Fix a line $R$ and assume that $A$ does not satisfies the G-condition for $A$ with respect to $R$, i.e. that $\operatorname{deg}(A \cap R) \geq$ $d$. Since $A \subseteq Z$, we get $\operatorname{deg}(Z \cap R) \geq d$. First assume $\operatorname{deg}(Z \cap R)>d$. Since $d+1 \geq \operatorname{deg}(Z \cap L) \geq \operatorname{deg}(Z \cap R)$, we get $\operatorname{deg}(Z \cap R)=\operatorname{deg}(Z \cap L)=d+1$ and hence (since $\sharp(S \cap R) \leq 2$ and $\sharp(S \cap R) \geq 2) m_{1}+m_{2}=d+1$ and

Now assume $\operatorname{deg}(Z \cap R)=d$, i.e. $S \cap D \cap R=\emptyset$. Assume for the moment $\sharp(S \cap R)=1$, say $S \cap R=\{u\}$. We have $k_{u}=d \geq m_{1}$. Hence $m_{1}=d$. Since $u_{Z}\left(\mathbb{P}^{2}\right)=2 d+2$ and $d \geq 3$, we have $m_{3}<d$. Hence $u \in D$ and $\left(k_{u}, e_{u}\right) \neq(1,1)$. Therefore $u_{A}(R)<u_{Z}(R)=d$, a contradiction. Therefore $\sharp(S \cap R)=2$. Let $\left\{o_{1}, o_{2}\right\}$ denote the points of $S \cap R$ with $m_{o_{1}} \geq m_{o_{2}}$. Since $m_{o_{1}}+m_{o_{2}}=d$, we have $m_{o_{1}} \geq\lceil d / 2\rceil$. Since $o_{1} \notin S \cap D$, we have $m_{o_{1}} \leq m_{6}$. Hence $2 d+2 \geq 6\lceil d / 2\rceil$, a contradiction.

Now we check when $A$ satisfies condition $\diamond$ in degree $d-2$. Fix $q \in S$ whose multiplicity in $A$ is maximal. First assume $q \notin D$ and hence $t_{Z, q}=t_{A, q}$. If $k_{q} \geq d-2$, the choice of $m_{1}, \ldots, m_{5}$ and $D$ gives $2 d+2 \geq 6(d-2)$, contradicting the assumption $d \geq 3$. Now assume $q \in S$. We have $t_{A, q}=\left(k_{q}-1, e_{q}\right)$ unless $t_{Z, q}=(1,1)$ and hence $t_{A, q}=(1,0)$. Since $d-2>0$, the latter case gives no obstruction to $\diamond$. Now assume that $k_{q}-1 \geq d-2$. We may assume $k_{q} \leq d$. First assume $k_{q}=d$. In this case we use the linear projection from $q$ to see that we are either in case (2) or in case (3) with respect to the point $q \in S$. 
Now assume $\left(k_{q}, e_{q}\right)=(d-1,0)$. In this case $q$ satisfies $\diamond$ for $A$ in degree $d-2$. Now assume $\left(k_{q}, e_{q}\right)=(d-1,1)$. Set $Z^{\prime}:=Z \backslash Z_{q}, Z^{\prime \prime}:=Z^{\prime} \cup(d-1) q$ and $Z^{+}:=Z^{\prime} \cup d q$. We have $u_{Z^{\prime \prime}}\left(\mathbb{P}^{2}\right)=2 d+1$. Since $Z^{\prime \prime} \subset Z^{\prime}$ and $Z$ satisfies $\diamond$ and the G-conditions in degree $d$ for lines, $Z^{\prime \prime}$ satisfies $\diamond$ and the G-conditions in degree $d$ for lines, Theorem 1 gives $h^{1}\left(\mathcal{I}_{Z^{\prime \prime}}(d)\right)=0$. Since $h^{1}\left(\mathcal{I}_{Z}(d)\right)>0$ and $\operatorname{deg}(Z)=\operatorname{deg}\left(Z^{\prime}\right)+1$, we get $H^{0}\left(\mathcal{I}_{Z}(d)\right)=h^{0}\left(\mathcal{I}_{Z^{\prime}}(d)\right.$. Since $Z_{q}$ is general in $W(q, d-1,1)$, we get $H^{0}\left(\mathcal{I}_{Z^{+}}(d)\right)=H^{0}\left(\mathcal{I}_{Z^{\prime}}(d)\right.$. We have $u_{Z^{\prime}}\left(\mathbb{P}^{2}\right)=u_{Z}\left(\mathbb{P}^{2}\right)-d=d+2$, i.e. $\sum_{u \in S \backslash\{q\}} k_{u}+e_{u}=d+2$. Let $\mu: \mathbb{P}^{2} \backslash\{q\} \rightarrow \mathbb{P}^{1}$ be the linear projection from $q$. Since $\sharp(S \cap T)=2$ for all lines $T, \mu \mid S \backslash\{q\}$ is injective. For each $u \in S \backslash\{q\}$ let $Z_{u}$ the connected of $Z$ containing $u$. $Z_{q}$ is the connected component of $Z^{\prime}$ containing $u$. Since each $Z_{u}$ is general in $W\left(u, k_{u}, e_{u}\right)$, the scheme $\mu\left(Z_{q}\right)$ has degree $k_{u}+e_{u}$. Since $\mu \mid S \backslash\{q\}$ is injective and $\sum_{u \in S \backslash\{q\}} k_{u}+e_{u}=d+2$, we have $h^{0}\left(\mathbb{P}^{1}, \mathcal{I}_{\mu\left(Z^{\prime}\right)}(d)\right)=0$. Hence $h^{0}\left(\mathcal{I}_{Z^{+}}(d)\right)=0$ and so $h^{0}\left(\mathcal{I}_{Z^{\prime \prime}}(d)\right)=0$. Since $h^{1}\left(\mathcal{I}_{Z^{\prime \prime}}(d)\right)=0$, we get $\operatorname{deg}\left(Z^{\prime \prime}\right)=\left(\begin{array}{c}d+2 \\ 2\end{array}\right)$. We have $\operatorname{deg}\left(Z^{\prime \prime}\right)=\left(\begin{array}{l}d \\ 2\end{array}\right)+\sum_{u \in S \backslash\{q\}}\left(\left(\begin{array}{c}k_{u}+1 \\ 2\end{array}\right)+e_{u}\right)$. Since $k_{u} \leq 2$ for all $u$, we have $\left.\left(\begin{array}{c}k_{u}+1 \\ 2\end{array}\right)+e_{u}\right) \leq 3\left(\sum_{u \in S \backslash\{q\}} k_{u}+e_{u}\right) \leq 3(d+2) / 2$, with equality if and only if $e_{u}=0$ and $k_{u}=2$ for all $u$. Hence $\left(\begin{array}{c}d+2 \\ 2\end{array}\right) \leq\left(\begin{array}{l}d \\ 2\end{array}\right)+3(d+2) / 2$, a contradiction if $d \geq 5$. Now assume $d=4$. It remains to handle only the case $\sharp(S)=3$ and $\left(k_{u}, e_{u}\right)=(2,0)$ for all $u \in S \backslash\{q\}$. In this case we have $h^{0}\left(\mathcal{I}_{Z^{\prime \prime}}(4)\right)=3$ and $h^{0}\left(\mathcal{I}_{Z^{\prime}}(4)\right) \geq 5$.

(ii) Now assume $\sharp(S \cap L)>2$. Since $u_{B}\left(\mathbb{P}^{2}\right)=u_{Z}\left(\mathbb{P}^{2}\right)-\sharp(S \cap L), B$ satisfies the $\mathrm{G}$-condition in degree $d-1$ with respect to $d-1$. First assume that $B$ does not satisfies $\diamond$ in degree $d-1$, i.e. there is $p \in S$ with $t_{B, p}=(d, 1)$. Since $Z$ satisfies $\diamond$ in degree $d$, we have $p \notin L$. Fix any $q \in S \backslash\{q\}$ and let $D$ be the line spanned by $p$ and $q$. We have $u_{Z}(D)>d$. The maximality property of $L$ gives $\operatorname{deg}(Z \cap L)=d+1$. Since $w\left(\mathbb{P}^{2}\right) \geq d+1+\operatorname{deg}(Z)+\sum_{q \in S \cap L} e_{q}$ with strict inequality if $S \backslash\{p\} \nsubseteq L$, we get $S \backslash\{p\} \subset L$ and $e_{q}=0$ for all $q \neq p$, i.e. we are in the second case listed in Theorem 2. Now assume that $B$ satisfies $\diamond$ in degree $d-1$. Since $B$ satisfies $\diamond$ and the G-condition in degree $d-1$ with respect to $\mathbb{P}^{2}$ and $h^{1}\left(\mathcal{I}_{B}(d-1)\right)>0$, it does not satisfies the G-condition for some line $D$, i.e. $\operatorname{deg}(D \cap B) \geq d+1$. The maximality property of $L$ gives $\operatorname{deg}(L \cap Z) \geq d+1$. Since $u_{Z}\left(\mathbb{P}^{2}\right)=2 d+2$, we get $S \subset R \cup L$ and $e_{a}=0$ for all $a \in S$, i.e. we are in case (1) with a reducible conic; the point $L \cap R$ is not in $S$, because we also get $\operatorname{deg}(R \cap B)=\operatorname{deg}(R \cap Z)$. 


\section{Acknowledgements}

The author was partially supported by MIUR and GNSAGA of INdAM (Italy).

\section{References}

[1] E. Ballico, On the homogeneous ideal of fat points: the Segre bound, preprint.

[2] E. Ballico, O. Dumitrescu and E. Postinghel, In preparation.

[3] B. Benedetti, G. Fatabbi and A. Lorenzini, Segre's bound and the case of $n+2$ fat points of $\mathbb{P}^{n}$, Comm. Algebra 40 (2012), 395-403.

[4] M. V. Catalisano, Linear systems of plane curves through fixed "fat " points of $\mathbf{P}^{2}$. J. Algebra 142 (1991), no. 1, 81-100.

[5] M. V. Catalisano, N. V.Trung and G. Valla, A sharp bound for the regularity index of fat points in general position. Proc. Amer. Math. Soc. 118 (1993), no. 3, 717-724.

[6] G. Fatabbi and A. Lorenzini, On a sharp bound for the regularity index of any set of fat points. J. Pure Appl. Algebra 161 (2001), no. 1-2, 91-111.

[7] P. V. Thiên On Segre bound for the regularity index of fat points in $\mathbb{P}^{2}$. Acta Math. Vietnam. 24 (1999), no. 1, 75-81.

[8] P. V. Thiên, Segre bound for the regularity index of fat points in $\mathbb{P}^{3}$. J. Pure Appl. Algebra 151 (2000), no. 2, 197-214

[9] N. C. Tu and T. M. Hung, On the regularity index of $n+3$ almost equimultiple fat points in $\mathbb{P}^{n}$. Kyushu J. Math. 67 (2013), no. 1, 20-213. 
